Abstract E-131 Table 1 Differences in Bolus arrival times after Pipeline placement and before Pipeline placement using two points (one just distal to the aneurysm being treated and one just proximal to the aneurysm being treated)

\begin{tabular}{llllll}
\hline RECON 1 & RECON 1 & RECON & RECON & RECON & RECON \\
Before & After & 2Before & 2After & 3Before & 3After \\
$0.10 \mathrm{~s}( \pm .13)$ & $0.09 \mathrm{~s}( \pm 0.56)$ & $0.08 \mathrm{~s}( \pm 0.13)$ & $0.09 \mathrm{~s}( \pm 0.13)$ & $0.14 \mathrm{~s}( \pm 0.20)$ & $0.16 \mathrm{~s}( \pm 0.33)$ \\
\hline
\end{tabular}

Abstract E-131 Table 2 Differences in Bolus arrival time after Pipeline placement and before Pipeline placement using two points (one just distal to the aneurysm being treated and one within the aneurysm being treated)

\begin{tabular}{lccccc}
\hline RECON 1 & RECON & RECON & RECON & RECON & RECON \\
Before & 1After & 2Before & 2After & 3Before & 3After \\
$0.046 \mathrm{~s}( \pm .14)$ & $0.08 \mathrm{~s}( \pm 0.28)$ & $0.10 \mathrm{~s}( \pm 0.31)$ & $0.01 \mathrm{~s}( \pm 0.12)$ & $0.15 \mathrm{~s}( \pm 0.33)$ & $-0.04 \mathrm{~s}( \pm 0.27)$ \\
\hline
\end{tabular}

The bolus arrival time was then measured at a point just distal to the aneurysm being treated and within the aneurysm itself (table 2). The RECON 1 protocol appeared to most reliably demonstrate slowed contrast inflow into the aneurysm post deployment of the Pipeline device.

Conclusion The 3D iFlow information obtained using 4D DSA imaging can provide clinicians information on the amount of flow pre and post deployment of a pipeline device for treatment of an intracranial aneurysm. This information could be useful to model aneurysm treatment and time to obliteration when utilizing pipeline flow diversion for the treatment of intracranial aneurysms. Continued work to refine protocols and software are needed to implement into clinical care.

Disclaimer This product is under development and not commercially available. Its future availability cannot be ensured.

Disclosures N. Khan: None. J. DiNitto: 5; C; Siemens. A. Birkhold: 5; C; Siemens. A. Arthur: None. C. Nickele: None. D. Hoit: None. V. Inoa: None. L. Elijovich: None.

\section{E-132 MICROPHONE ENABLED CATHETER TO QUANTIFY VENOUS PULSATILE TINNITUS IN PATIENT-SPECIFIC FLOW MODELS}

M Amans*, K Valluru, S Kondapavulur, B Kilbride, H Haraldsson, W Smith, K Meisel, D Saloner. Radiology and Biomedical Imaging, University of California San Francisco, San Francisco, CA

\subsection{6/neurintsurg-2020-SNIS. 164}

Purpose Pulsatile tinnitus (PT) is a debilitating condition that can be caused by abnormal blood flow in venous vasculature near the cochlea ${ }^{1}$. Often times PT is very difficult to diagnose reliably with diagnosis rates typically hovering around $50 \%$ and requiring multiple advanced imaging procedures including diagnostic cerebral angiography ${ }^{2}$. A tool that could objectively mimic a PT patient's hearing experience would allow physicians to reliably diagnose the cause of this difficult symptom.

Materials and Methods We developed a prototype of the 'Phonocatheter', a 6 Fr catheter that can measure and record PT sounds as well as replay them in real time. The Phonocatheter consists of an embedded microphone at its distal end to record intravascular sounds and relay them to a microcontroller (Arduino Mega 2560 Rev3) based custom data acquisition interface. Two benchtop PT flow models ${ }^{3}$ that represent a PT patient's transverse sinus (TS) anatomy before and after lumbar puncture (LP) were created and used to test the Phonocatheter. Glycerol-water mixture was pumped through these models to mimic blood flow at a mean flow rate of $7.4 \mathrm{cc} / \mathrm{s}$. The Phonocatheter was inserted through a $9 \mathrm{Fr}$ access port and was navigated into the TS stenosis region (figure 1A). A handsfree Bluetooth compatible electronic stethoscope was placed externally over the same TS region to record PT transluminally and validate the sound measurements recorded by Phonocatheter $(n=10)$. The sound measurements acquired by Phonocatheter and stethoscope were saved in .wav file format and were exported to MATLAB for comparative analysis. Variation in peak-to-rms sound amplitude values from the TS in pre-LP and post-LP models was calculated. Wilcoxon rank sum test was used to statistically determine the differences in

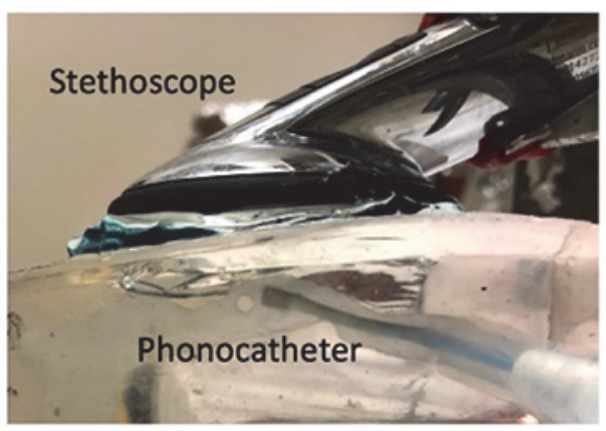

A

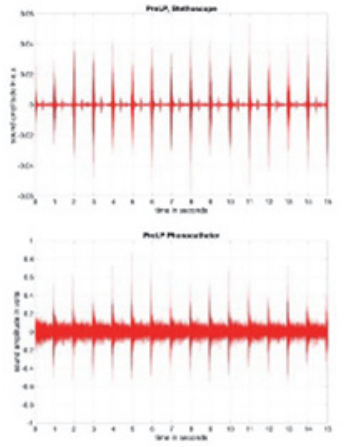

B

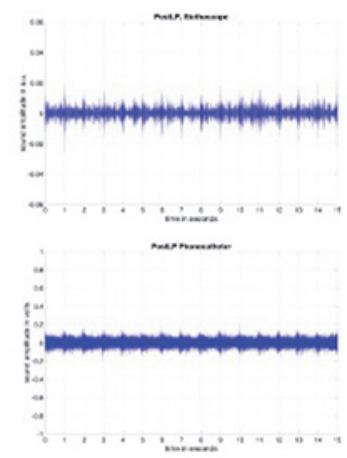

C

Abstract E-132 Figure 1 A) Phonocatheter and a handsfree electronic stethoscope recording sounds generated from transverse sinus stenosis in a patient specific benchtop model of Pulsatile Tinnitus. An external pump (not shown) simulating cardiac rhythm (60 beats per minute) is connected to the flow model to circulate glycerol-water mixture at a characteristic flow rate of $7.4 \mathrm{cc} / \mathrm{s}$. Ultrasound transmission gel was used to couple the stethoscope to the surface of the model. B) Sounds recorded from the model representing the transverse sinus anatomy with stenosis before lumbar puncture (preLP) with stethoscope (top) and phonocatheter (bottom) suggest that the sound was synchronous to the cardiac rhythm mimicking PT. C) Sounds recorded from the model representing the transverse sinus anatomy immediately after lumbar puncture (postLP) with Stethoscope (top) and phonocatheter (bottom) demonstrate that the sound amplitude was weaker compared to the preLP model. There was a reduction in stenosis 
sound measurements between the two patient-specific models for each sensor.

Results Both the Phonocatheter and stethoscope were able to record transluminal and intravascular sounds generated from stenosis respectively. The Phonocather was in good agreement with the stethoscope demonstrating that the peak-to-rms (mean \pm standard deviation) sound amplitude was significantly louder $(\mathrm{p}<0.0001)$ in the TS stenosis region in pre-lumbar puncture model (Stethoscope: $9.03 \pm 1.61$; Phonocatheter: $6.62 \pm 1.55)$ compared to the TS region in post-lumbar puncture model (Stethoscope: $4.20 \pm 0.86$; Phonocatheter: $3.62 \pm 0.88$ ) (figures $1 \mathrm{~B}$ and $1 \mathrm{C}$ ).

Conclusion We have developed a prototype of microphone enabled catheter that can measure sound in patient-specific PT flow models, and potentially measure sounds quantitively in PT patients.

Disclosures M. Amans: 1; C; NIH R01 HL149124-01A1. K. Valluru: None. S. Kondapavulur: None. B. Kilbride: None. H. Haraldsson: None. W. Smith: None. K. Meisel: None. D. Saloner: None.

\section{E-133 DELAYED INTRACEREBRAL HEMORRHAGE AFTER WINGSPAN STENTING FOR INTRACRANIAL INTERNAL CAROTID ARTERY STENOSIS: A CASE REPORT}

1J Kim*, ${ }^{2}$ CHUNG. 'Radiology, Gangnam Severance Hospital, Seoul, Korea, Republic of; ${ }^{2}$ Neurosurgery, Severance Hospital, Seoul, Korea, Republic of

10.1136/neurintsurg-2020-SNIS. 165

Acute ischemic infarction from intracranial atherosclerotic disease accounts for approximately $8 \%-10 \%$ of strokes in the United States each year. At a recent large prospective randomized trial, the efficacy and safety of wingspan stent for intracranial atherosclerotic stenosis was verified when it was used by experienced interventionalist with proper patient selection. Among several procedural complications, delayed hemorrhage is rarely reported. A 45-year-old man presented with a history of dysarthria and recurrent episodes of transient right hemiparesis. The patient was newly found atrial fibrillation and started warfarin medication. Cerebral catheter angiography and single-photon emission computed tomography (SPECT) scan with acetazolamide challenge confirmed moderate (60\%) left carotid artery stenosis and symmetrical findings of both hemispheres. Endovascular Wingspan

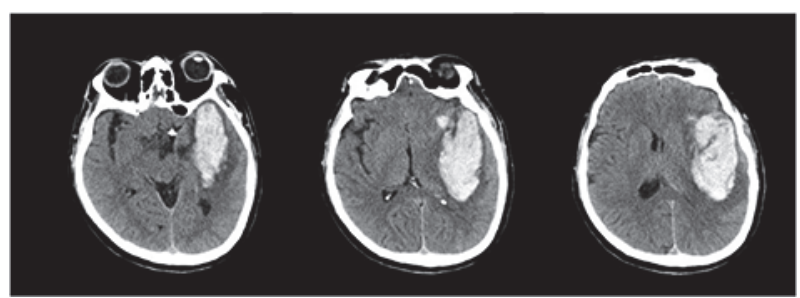

Abstract E-133 Figure 2

stenting of the left communicating segment of internal cerebral artery was performed uneventfully. 22 days after treatment the patient presented with sudden headache and aphasia and CT scan showed intracerebral hemorrhage on left temporal lobe. Due to further rapid clinical deterioration, surgical removal of hematoma was required. The patient's poor neurological outcome did not improve during the 2year follow-up period. This case report illustrates a severe delayed intracerebral hemorrhage following intracranial stenting of the internal carotid artery. The mechanism of delayed hemorrhage is unclear. Currently, 4 mechanisms have been discussed as potential causes of remote ICH after uneventful EVT. These mechanisms are dual antiplatelet therapy, hemorrhagic transformation of clinically silent small periprocedural embolic infarcts, intraprocedural foreign body emboli, and flow modification. This complication should not be neglected because of its poor clinical outcomes.

Disclosures J. Kim: None. J. Chung: None.

\section{E-134 TRANS-ULNAR ACCESS FOR NEUROINTERVENTION: PUSHING THE BOUNDARIES OF ARTERIAL ACCESS}

R Dossani, M Waqas*, M Tso, G Rajah, F Chin, A Yunke, A Siddiqui, J Davies. UB Neurosurgery, Buffalo, NY

\subsection{6/neurintsurg-2020-SNIS. 166}

Background Transradial access has been gaining popularity in neurointerventional surgery. Failure to obtain radial access may lead neurointerventionists to convert to a transfemoral approach, which is associated with a higher complication rate than the radial approach. Ulnar access, however, provides a similar safety profile to radial access and permits neurointerventionists to work in the same hand without having to convert to a femoral approach. In this study, we evaluate the feasibility of using transulnar access to perform diagnostic cerebral angiograms and various neurointerventional procedures.

Methods Consecutive patients who underwent transulnar diagnostic angiogram or neurointerventional procedures were included in the study over a period of 12 months. Data on demographics, procedure indication, devices, technique and complications recorded. A descriptive analysis was carried out.

Results Transulnar access was utilized in 18 patients over the study period. Mean age was $71.6+6.8$ years; $10(55.5 \%)$ patients were male. Fifteen diagnostic angiograms and 3 neurointerventions (1 left middle meningeal artery, 1 right carotid artery stenting, 1 left carotid artery stenting) were performed. All the procedures were performed using a right sided ulnar artery with ultrasound guidance. The indications for ulnar access included a feeble radial artery pulse $(n=17)$, or radial 